\title{
The Effectiveness of UV-LED Photopolymerisation over Conventional UV-Mercury for Polyurethane Acrylate Coating
}

\author{
Siti Khairunisah Ghazali, Fatria Syaimima Syaiful Azim, Nadia Adrus, and \\ Jamarosliza Jamaluddin* \\ Department of Bioprocess and Polymer Engineering, School of Chemical and Energy \\ Engineering, Faculty of Engineering, Universiti Teknologi Malaysia, \\ 81310 Johor Bahru, Johor, Malaysia \\ *jamarosliza@utm.my
}

\begin{abstract}
This paper presents the effect of UV-LED and UV-mercury as the light source toward curing behaviour of urethane acrylate coating. The UV-curable coating was prepared based on aliphatic urethane acrylate oligomer, 2-ethylhexyl acrylate (2-EHA), methyl methacrylate (MMA), trimethylolpropane triacrylate (TMPTA), and commercial photoinitiator. The effect of irradiation time on curing behaviour was investigated using Fourier Transform Infra-Red (FTIR) and the percentage of $\mathrm{C}=\mathrm{C}$ conversion was calculated. From the ATR-FTIR spectra, the $\mathrm{C}=\mathrm{C}$ absorption peak at $810 \mathrm{~cm}^{-1}$ and $1635 \mathrm{~cm}^{-1}$ was found to be decreased after UV irradiation, indicated the proceeding of the polymerisation reaction. In term of $\mathrm{C}=\mathrm{C}$ conversion, $\mathrm{UV}-\mathrm{LED}$ irradiation showed better results with $97-99 \%$ than UV-mercury irradiation which is $86-96 \%$. It was also observed that the tackiness of the coating reduced via UV-LED irradiation, suggesting higher conversion of monomers. Gel fraction of the coating also measured and higher gel fraction values, > $98 \%$ in this study was associated with higher cross-linking density. The results of this study depicted that UV-LED is a viable alternative to replace UV-mercury-based lamp in the coating industry.
\end{abstract}

Keywords: UV-LED, UV-mercury, Photopolymerisation, Urethane acrylate, Curing behaviour

\section{Introduction}

Photopolymerisation or light-induced polymerisation or also known as radiation curing is one of the techniques to promote green or environmental friendly technology in coating industry [1,2]. Historically, solvent-borne coating was the most employed method involving $50-70 \%$ the usage of solvent [3]. Solvent-borne coating demonstrates excellent properties of the coating and lowers operation cost. It is also easy to handle in terms of processing. Since health and environmental apprehension become an important and crucial concern in coating sectors, the development of coating products with minimal environmental impacts such as low emission of volatile organic compounds (VOCs), energy consumption reduction and toxicity continue to be in high demand. Therefore, UV radiation coating was a smart approach since it offers remarkable properties such as low energy consumption, high solid formulation (solvent-free formulation) and time efficient without compromising its desired coating performance.

Traditionally, UV-mercury (UV-Hg) lamp was extensively used as the light source for the radiation curing due to its broad wavelength distribution which easily matched the absorption of photoinitiator. However, it is often offset by the production of harmful ozone and mercury. Due to its toxic nature, there is an urge to eradicate its usage worldwide. According to the European Union's "Restriction of Hazardous Substance" directive or known as RoHS 2, the usage of UV-Hg lamp needs to be eliminated in 
all instances. In contrast for the curing purposes, they have been exempted up until July 22, 2016 and if the exemption is not extended, their usages will be banned especially in Europe [4]. Hence, in order to develop a green aspect in photopolymerisation, UV-LED has been utilised as a new alternative to the conventional UV curing [4-10].

UV LED photopolymerisation or curing refers to the method of curing by utilizing the energy output from the LED in UV spectrum. Elimination of harmful mercury and extraction of ozone makes the UV-LED system inherently environmentally friendly [11-17]. Besides that, LEDs longer lifetime, suitability for heat sensitive materials and the capability of instant on/off operation makes the UV LED system superior to existing UV mercury. In addition, UV-LED consumes $50 \%$ less energy than the equivalent mercury arc lamp due to its instant on/off capability as well as no unnecessary wavelength emitted (narrow wavelength distribution) $[8,13]$.

However, LEDs also have a few challenges to handle with such as limited matching photoinitiator (PI), UV chemistry formulation and surface cure issues [8,11-18]. Okamura et al. [9] reported that the UV-C LEDs $(265 \mathrm{~nm}, 285 \mathrm{~nm}$, and $300 \mathrm{~nm}$ ) can be effectively mitigate the oxygen inhibition in UV curing. Recently, Ayub et al. [19] successfully developed a new formulated hydrogel for UV-LED system with a modified water-soluble photoinitiator. The hydrogel conversion reported was as high as $90 \%$. Landry et al. [8] found that the conversion of high-solid formulation for wood coating when exposed to UV-LED irradiation was low $(<70 \%)$ compared to water-based formulation as the conversion achieved was $>90 \%$.

As the exploitation of the UV-LED technology has been intensified in recent years, this paper presents a suitable high-solid coating formulation for UV-LED photopolymerisation. Since different light or irradiation profiles can produce different properties of UV cured coating, this paper presents the effect of UV-LED and UV-Hg as the light source toward curing behaviour of the urethane acrylate coating. The feasibility of UV-LED to replace current UV-Hg was investigated in terms of degree of conversion using Fourier Transform Infra-Red (FTIR) and gel fraction measurement.

\section{Experimental}

Aliphatic urethane acrylate oligomer (BOMAR ${ }^{\mathrm{TM}}$ 7432-GB) was generously obtained from Dymax Corporation while 2-ethylhexyl acrylate (2-EHA), methyl methacrylate (MMA)

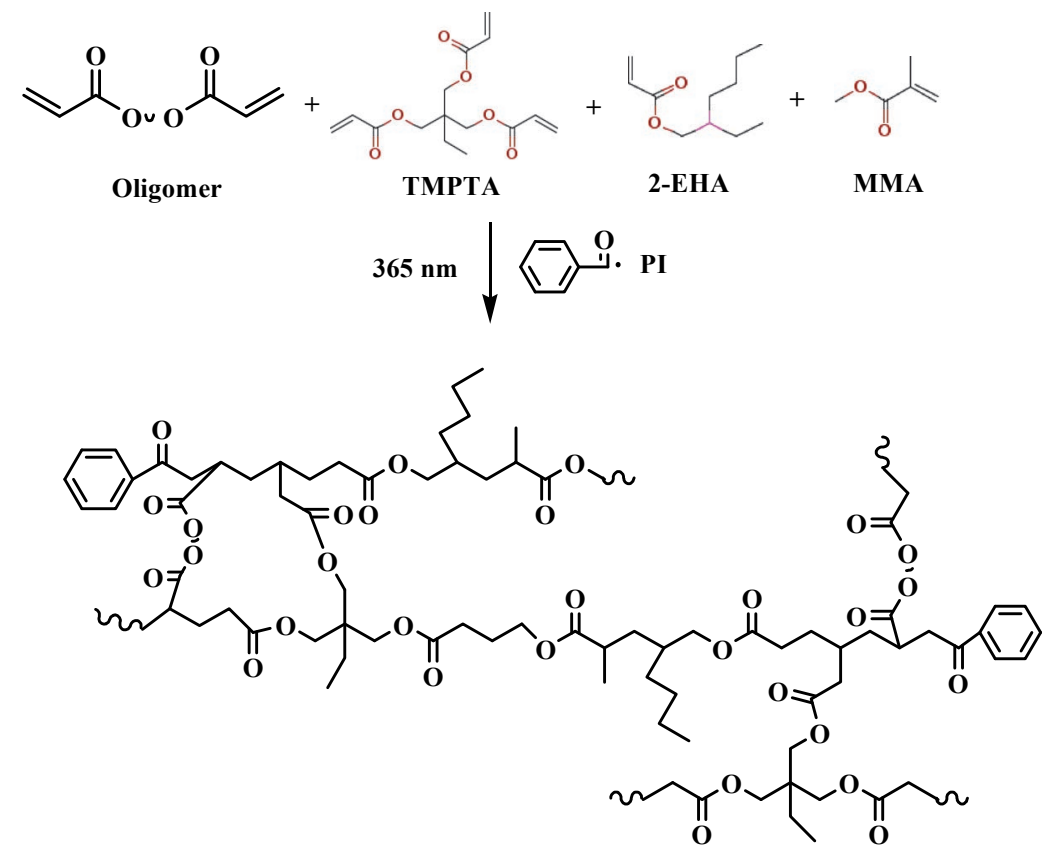

Curable urethane acrylate

Scheme 1. Reaction mechanism of photopolymerisation in UV-LED irradiation at $365 \mathrm{~nm}$. 
and trimethylolpropane triacrylate (TMPTA) were purchased from Sigma Aldrich (Malaysia). The commercial photoinitiator oligo[2-hydroxy-2-methyl-1-[4-1(1-methylvinyl) phenyl]propanone] (Chivacure ${ }^{\circledR} \quad 300$ ) was obtained from Chitec. All the chemicals were used without further purification. Chemical structures of the compounds are included in Scheme 1.

The UV-curable coating formulation was prepared by dissolving photoinitiator in the reactive monomers for an hour at room temperature. Then, the oligomer was added into the mixture and was stirred for another an hour. The prepared formulation as shown in Table 1 was coated onto the glass substrate $(10 \mathrm{~cm} \times 5$ $\mathrm{cm} \times 0.3 \mathrm{~cm}$ ) with the thickness of $50 \mu \mathrm{m}$ using a bar applicator. The wet coating was exposed to UV irradiation (Hönle UV-LED chamber and Hönle UV-Hg chamber) with a wavelength of $365 \mathrm{~nm}$ and $15 \mathrm{~cm}$ from lamp to the sample by varying the irradiation time from 0 to $30 \mathrm{~min}$. The light intensity of the UV-LED was

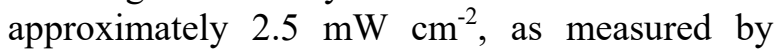
Hönle UV meter.

In order to analyse the chemical changes in the cured sample, the disappearance of functional group was monitored by using attenuated total reflectance mode FTIR (Shimadzu IR Tracer 100). FTIR spectra were obtained in the $4 \mathrm{~cm}^{-1}$ resolution mode with 32 scans averaged for each sample between $4000 \mathrm{~cm}^{-1}$ and $400 \mathrm{~cm}^{-1}$ at room temperature.

In this study, conversion of the acrylate was calculated using the following equation [20]:

$$
\text { Conversion }(\%)=\frac{\left(A_{0}-A_{t}\right)}{A_{0}} \times 100 \%
$$

where $A_{0}$ is the peak area of $802-817 \mathrm{~cm}^{-1}$, including the absorption peak at $810 \mathrm{~cm}^{-1}$ before $\mathrm{UV}$ exposure and $A_{\mathrm{t}}$ is the area at UV exposure time $t$. The area at peak $810 \mathrm{~cm}^{-1}$ was selected because the changes of the unsaturation absorbance in the UV-curable acrylate based coating is easily observed [21].

Gel fraction measurements were determined by soaking the sample in toluene for 1 day at room temperature. The UV-curable film was filtered and dried at $50{ }^{\circ} \mathrm{C}$ until its weight is constant. The gel fraction of the UV-cured film was calculated by the equation below [20-22]:

$$
\text { Gel Fraction }(\%)=\frac{w_{1}}{w_{0}} \times 100 \%
$$

where $w_{0}$ is weight of the cured sample and $w_{1}$ is the weight of the cured sample (weight after filtration and drying).
The tackiness of the cured coating was examined according to the cure grade measurement and determined by touch [17]. The cured surface was assessed based on: 0-wet surface; 1- surface sticks $\mathrm{t}$ finger; 2- surface slightly sticks to finger and 3- surface does not stick to finger.

Table 1. Formulation of the urethane acrylate coating.

\begin{tabular}{ccc}
\hline \multirow{2}{*}{ Components } & \multicolumn{2}{c}{ Composition (wt\%) } \\
\cline { 2 - 3 } & UV-LED & UV-Hg \\
\hline BOMAR $^{\text {TM }}$ 7432-GB Oligomer & 70 & 70 \\
MMA & 13 & 13 \\
2-EHA & 8 & 8 \\
TMPTA & 5 & 5 \\
PI & 4 & 4 \\
\hline
\end{tabular}

\section{Results and discussion}

\subsection{FTIR measurement}

Figure 1 illustrates the FTIR spectra of the UV-curable coating before and after UV irradiation. As shown in Fig. 1, the absorption band at peak $810 \mathrm{~cm}^{-1}$ and $1635 \mathrm{~cm}^{-1}$ decreased respectively after UV irradiation, which is attributable to the gradual disappearance of the $\mathrm{C}=\mathrm{C}$ double bond. In fact, it is also found that the intensity of the related peaks decreased with the increase of irradiation time implying the consumption of $\mathrm{C}=\mathrm{C}$ double bond throughout the photopolymerisation [8].

The degree of conversion is determined by the proportion of the remaining concentration of the aliphatic $\mathrm{C}=\mathrm{C}$ in a cured sample relative to the total number of $\mathrm{C}=\mathrm{C}$ in the uncured material. According to the previous studies [20-23], the decreasing in the peak area at around $810 \mathrm{~cm}^{-1}$ and $1635 \mathrm{~cm}^{-1}, \mathrm{CH}_{2}=\mathrm{CH}$ - twisting and $\mathrm{CH}_{2}=\mathrm{CH}$ stretching respectively, indicated the degree of curing or the degree of double bond conversion.

In this paper, the degree of conversion was investigated in the functions of UV irradiation time and the effect of UV light sources; LED and $\mathrm{Hg}$ lamp, respectively. The results obtained were depicted in Fig. 2 and the conversion sharply increased up to approximately $97-99 \%$ within five minutes, but thereafter the conversion became constant with further increase of the irradiation time. However, $\mathrm{C}=\mathrm{C}$ conversion for UV-Hg lamp showed lower values ranging from $85-97 \%$ throughout the photopolymerisation. It 


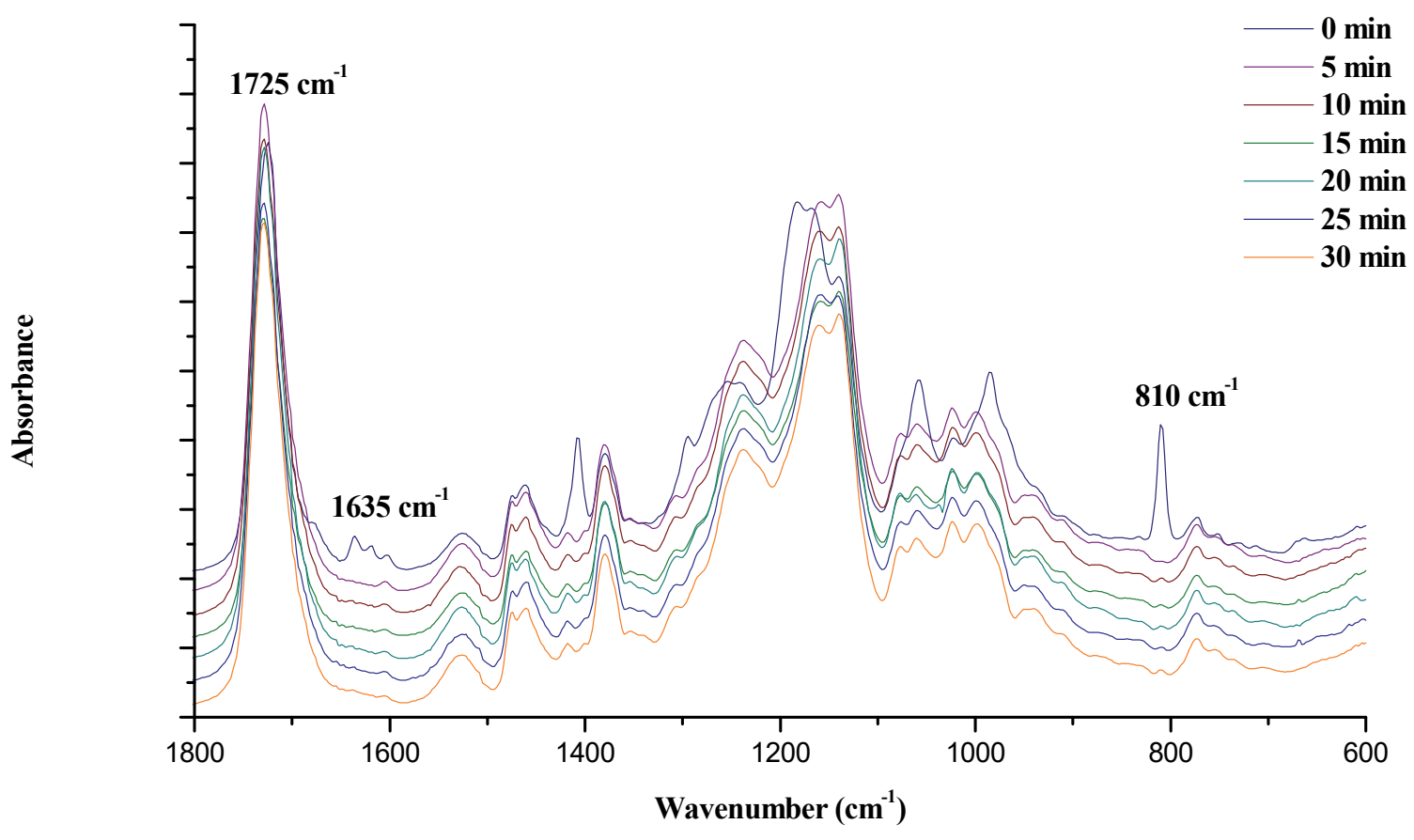

Fig. 1. FTIR spectra of urethane acrylate coating at different irradiation time for UV-LED photopolymerization.

Table 2. Effect of irradiation time on the degree of conversion and its tackiness.

\begin{tabular}{ccccc}
\hline \multirow{2}{*}{ Irradiation time (min) } & \multicolumn{2}{c}{ Conversion (\%) } & \multicolumn{2}{c}{ Cure grade $^{\mathrm{a}}$} \\
\cline { 2 - 5 } & UV-LED & UV-Hg & UV-LED & UV-Hg \\
\hline 0 & 0 & 0 & 0 & 0 \\
5 & 97 & 87 & 1 & 1 \\
10 & 99 & 93 & 2 & 1 \\
15 & 98 & 94 & 2 & 1 \\
20 & 98 & 96 & 2 & 2 \\
25 & 98 & 95 & 3 & 2 \\
30 & 98 & 96 & 3 & 2 \\
\hline
\end{tabular}

a $0=$ Wet surface; $1=$ Surface sticks to finger; $2=$ Surface slightly sticks to finger; $3=$ Surface does not stick to finger (tack-free) [15].

was suggested that the UV-Hg did not reach similar final $\mathrm{C}=\mathrm{C}$ conversion as the UV-LED system because the photoinitiator used may be more susceptible towards the wavelength emitted by the UV-LED than UV-Hg lamp system [24].

In addition, UV-LED also has the advantage to emit specific and narrow wavelength with more concentrated energy since it has a monochromatic peak $[14,17,24]$ and almost $96 \%$ of the energy emitted enhanced the absorption of the photoinitiator [8]. Conversely, conventional arc lamps such as mercury usually presents broader peak distribution throughout the UV spectrum and it may lead to the unwanted side reaction. This system could explain why UV-LED presents higher conversion than UV-Hg in this work.

A tack-free surface coating is crucial, and Table 2 presents the effect of the irradiation time on the tackiness of the cured sample. It is observed that the tackiness of the surface reduced with the increase of the irradiation time for both UV-LED and UV-Hg system. However, the UV-LED system exhibits an efficient photopolymerisation compared to the UV-Hg system as a tack-free surface obtained after 25 minutes of photopolymerisation. Meanwhile, the 


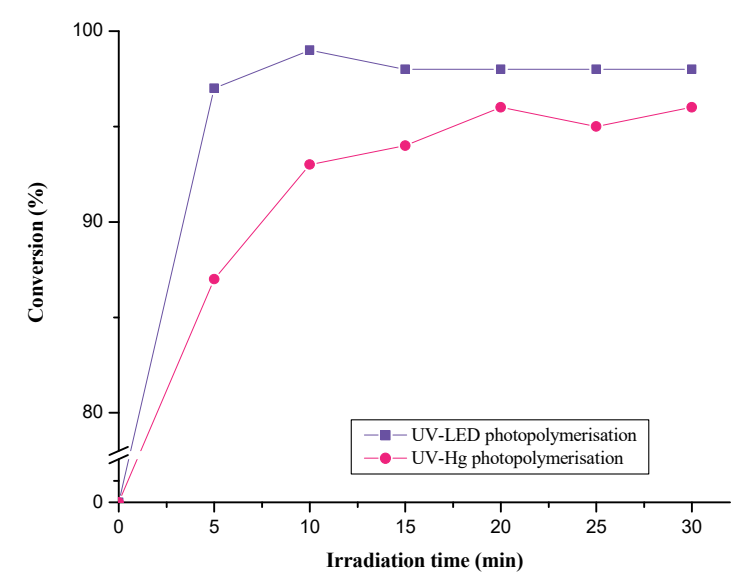

Fig. 2. Degree of conversion of the urethane acrylate coating for both UV-LED and UV-Hg at different irradiation time.

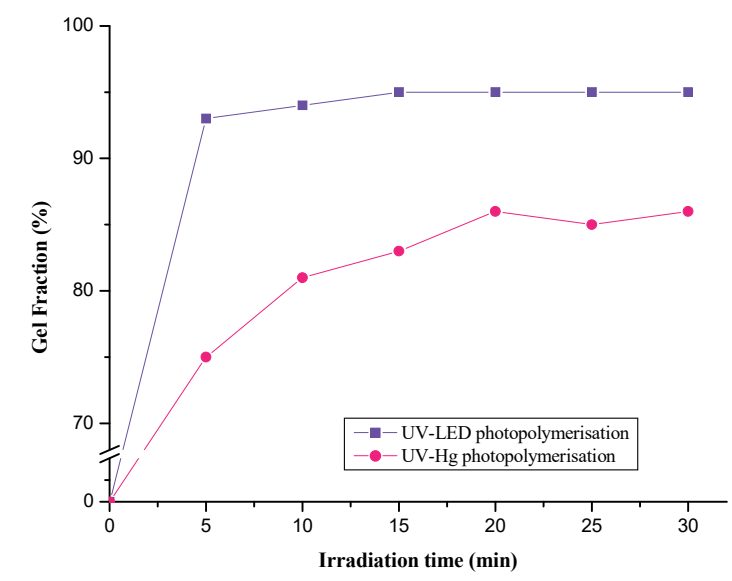

Fig. 3. Gel fraction of the urethane acrylate coating for both UV-LED and UV-Hg at different irradiation time.

surface of the coating for UV-Hg system did not achieve a tack-free surface even after 30 minutes of photopolymerisation. This finding can be relatable as the degree of conversion for the UV-Hg system (87-96\%) is lower than the UV-LED system (97-99\%). Therefore, the unreacted monomers remained in the system led to a tacky surface coating $[8,17]$.

\subsection{Gel fraction}

Gel fraction was measured to determine the degree of crosslinking of the curable coating by calculated the insoluble part of the crosslinked and network polymer [25]. Figure 3 shows the change of gel fraction as a function of UV irradiation time for both UV-LED and UV-Hg photopolymerisation system. It is observed that there are dramatic changes in gel fraction upon five minutes of UV irradiation. In the early stage of the photopolymerisation, the gel fraction increased rapidly because the lower viscosity of the system before UV-irradiation allowed the molecules and polymer chain to move freely and the double bond density was high, thus fast photopolymerisation occurred. Thus, led to increasing gel fraction [25]. This trend also indicated that the coating started to crosslink upon UV irradiation. In addition, it can be postulated that the presence of tri-functional acrylate monomer, TMPTA in the system accelerated the formation of the cross-linking network as illustrated in Scheme $1[21,22,26]$.

However, the gel fraction values almost plateau throughout 30 minutes of UV irradiation and it reached as high as $95 \%$ for UV-LED photopolymerisation. Meanwhile, for UV-Hg photopolymerisation, it showed lower gel fraction and achieved the highest, approximately $86 \%$ after 30 minutes. The slow and plateau trend of the gel fraction after 10-15 minutes of $\mathrm{UV}$-irradiation is due to the depletion of double density and all the monomer almost used throughout the photopolymerisation and forming a crosslinked network. Obviously, these results were in good agreement with the results of the $\mathrm{C}=\mathrm{C}$ conversion reported in the previous section. It is possible that some growing polymer and monomer trapped in the network. Since the crosslinked polymer will increase the viscosity of the system, it will restrict the mobility of the growing polymer and hindered them for further reaction, which explained why the conversion and gel fraction did not achieve $100 \%[27,28]$.

\section{Conclusion}

A suitable formulation of urethane acrylate coating for UV-LED photopolymerisation system was obtained. The feasibility of the UV-LED system as a new alternative for conventional $\mathrm{UV}-\mathrm{Hg}$ system was carried out in terms of the degree of conversion and gel fraction measurement. The comparisons were made in the function of irradiation time. It was found that the UV-LED photopolymerisation system demonstrated higher degree conversion $(\leq 99 \%$ vs. $\leq 96 \%)$ and higher gel fraction $(\leq 93 \%$ vs. $\leq$ $86 \%$ ). A tack-free surface coating also achieved after 25 minutes when the UV-LED system was 
adopted. From this finding, UV-LED appears reliable to replace current $\mathrm{UV}-\mathrm{Hg}$ system with promising features such as energy-efficient and environmentally friendly choice with the elimination of mercury-dependent source.

\section{Acknowledgement}

The authors thank the Ministry of Higher Education Malaysia and Research Managing Centre, Universiti Teknologi Malaysia for managing the grant (FRGS vote no: 4F960).

\section{References}

1. M. Tehfe, F. Louradour, J. Lalevée, and J. P. Fouassier, Appl. Sci., 3 (2013) 490.

2. C. Decker, Macromol. Rapid Commun., (2002) 1067.

3. R. Schwalm, Elsevier, $\mathbf{1}^{\text {st }} \mathbf{E d}$ (2007) 1.

4. T. R. Mawby, (2016) 1. Available from: http://uvledcommunity.org/uploads/pdfs/UVLED-NPIRI-Paper_FlintGroup_Dec2014.pdf.

5. M. Hubert and C. Dimas, Appl. Photonic Technol., 5260 (2003) 163.

6. R. Xie and D. Li, Opt. Laser Technol., 44 (2012) 1163.

7. H. J. Mehnert, R., Salleh, N. G., Alias, and M. S. Glasel, Radiat. Phys. Chem., 84 (2013) 70.

8. V. Landry, P. Blanchet, G. Boivin, J. F. Bouffard, and M. Vlad, Coatings, 5 (2015) 1019.

9. H. Okamura, S. Niizeki, T. Ochi, and A. Matsumoto, J. Photopolym. Sci. Technol., 29 (2016) 99.

10. K. Taki and K. Sawa, J. Photopolym. Sci. Technol., 31 (2018) 753.

11. T. R. Mawby, Radtech UV EB Conf., (2014) 1.

12. P. Mills, (2014). Available from: $\mathrm{http} / / /$ ledcoatings.com/files/Characterizing_th Output_and_performance_of_LEDs.pdf.

13. E. Kiyoi, RadTech Report, 2 (2013) 29.
Available from: http://radtechreport.com/ 42013_issue2_kiyoi.html.

14. R. Karsten, B. Larson, and K. Miller, (2014) 1. Available from: http://www.phoseon.com/ uploads/pdfs/documentation/characterizingthe-efficiency-of-uv-led-curing.pdf.

15. E. Kiyoi, RadTech Report, (2014) 29. Available from: http://www.radtech.org/ magazinearchives/Publications/RadTechRepo rt/jun-2014/Wood Coating with UV-LED Curing-A Focus on Heat.pdf.

16. B. Cortelyou, LEDs Magazine, 3 (2014) 1. Available from: http://www.ledsmagazine. com/articles/print/volume-11/issue-3/features/ science/uv-led-advancements-extend-the-pro mise-in-curing.html.

17. K. Anyaogu, L. Zhou, and J. Lalevée, J. Appl. Polym. Sci., 105 (2007) 803.

18. E. Kiyoi, Eur. Coat. J., (2013) 26.

19. N. F. Ayub, S. Hashim, J. Jamaluddin, N. Adrus, New J. Chem., 41 (2017) 5613.

20. B. H. Lee, J. H. Choi, and H. J. Kim, J. Coat. Technol. Res., 3 (2006) 221.

21. J. Jamaluddin and M. C. Lee, Prog. Polym. Rubber Technol., 812 (2013) 131.

22. D. Kunwong, N. Sumanochitraporn, and S. Kaewpirom, Songklanakarin J. Sci. Technol., 33 (2011) 201.

23. F. Li, S. Zhou, B. You, and L. Wu, J. Appl. Polym. Sci., 99 (2006) 1429.

24. S. L. McDermott, J. E. Walsh, and R. G. Howard, Opt. Laser Technol., 40 (2008) 487.

25. Y. J. Park, D. H. Lim, H. J. Kim, H. S. Joo, and H. S. Do, J. Adhes. Sci. Technol., 22 (2008) 1401.

26. X. Xiao and C. Hao, Colloids Surf. A, Physicochem. Eng. Asp., 359 (2010) 82.

27. L. Liao, H. Yue, and Y. Cui, Chinese $J$. Chem. Eng., 19 (2011) 285.

28. E. Andrzejewska, Prog. Polym. Sci., 26 (2001) 605 . 